\title{
Recovery of Hormone Sensitivity after Salvage Brachytherapy for Hormone Refractory Localized Prostate Cancer
}

\author{
Dan Smith, P. Nick Plowman \\ Department of Radiotherapy (DS, PNP), St Bartholomew's Hospital, West Smithfield, London and \\ Department of Radiotherapy (PNP), The Cromwell Hospital, Cromwell Road, London
}

\begin{abstract}
Purpose: Recent work has demonstrated the return of hormone sensitivity after palliative chemotherapy in androgen independent prostate cancer. We wished to establish whether a similar phenomenon existed in patients with no exposure to chemotherapy.

Materials and Methods: A review of "hormone resistant" patients who had received salvage brachytherapy for localized prostate cancer after previous external beam radiotherapy was undertaken. Three patients with subsequent biochemical relapse responded to the rechallenge with hormonal treatment.

Results: The series of patients presented here demonstrates this phenomenon occurs after salvage brachytherapy with no exposure to chemotherapy. Recovery of sensitivity is demonstrated both to androgen deprivation and to androgen receptor antagonism. The recovery of hormone sensitivity was surprisingly durable, ranging from eight months to over twenty-one months.

Conclusions: Hormone sensitivity may be recovered after salvage brachytherapy. Potential mechanisms underlying these observations are discussed and the likely central role of the activity of the androgen receptor highlighted. The relevance of these findings to the management of advanced prostate cancer is considered including thoughts on the practice of intermittent anti-androgen therapy.
\end{abstract}

Key words: prostatic neoplasm; androgen antagonists; brachytherapy; radiotherapy

Int Braz J Urol. 2010; 36: 283-91

\section{INTRODUCTION}

Prostate cancer is unique amongst malignancies in that its initial growth is dependent on the presence of intrinsic androgens. Whilst mutations in several tumor suppressor genes have been described and are thought important in the establishment of a clonal population of cells, development of clinically significant cancer also requires an androgenic drive to cellular proliferation (1).

This necessary androgenic drive provides therapeutic targets which may be exploited to inhibit the growth of prostate cancer. Recurrent or metastatic disease is typically first treated with hormonal manipulation: strategies include testicular androgen deprivation by either bilateral orchidectomy (2) or administration of a luteinizing hormone releasing hormone (LHRH) agonist (3), and treatment with anti-androgens such as flutamide to compete with testosterone for the androgen receptor binding site (4). Intrinsic androgenic drive is thus abrogated, apoptosis of tumor cells occurs and there is subsequent tumor regression with a fall in the plasma level of prostate specific antigen (PSA). 
Unfortunately, resistance to androgen suppression invariably develops: cells accumulate further genetic abnormalities and proliferate despite low testosterone levels at a median interval of 12-16 months after initiation of endocrine treatment (5).

Subsequent lines of hormonal manipulation act through related pathways and include the use of the synthetic estrogen diethylstilboestrol (6), the reduction of adrenal androgen production by administration of adrenocorticotropic hormone-suppressive glucocorticoids, e.g. hydrocortisone (7), and inhibition of the androgen synthesizing hormones 17-alpha hydroxylase and C17,20 lyase by abiraterone (8).

Conventional wisdom is that the loss of hormone sensitivity is a fixed, irreversible event, comparable to the loss of sensitivity to tamoxifen or an individual chemotherapeutic agent in breast cancer. Once the range of hormonal options is exhausted there is thought no benefit to restarting hormonal treatments to which the cancer has previously exhibited resistance.

However, the recovery of sensitivity of prostate cancer to LHRH agonist and to diethylstilboestrol has recently been reported following palliative CL56 (chlorambucil/ lomustine) chemotherapy (9) despite prior acquired resistance to both primary androgen suppression and estrogen therapy. It was postulated that the chemotherapy may have altered the subsequent behavior of the disease, particularly as a large proportion of patients with second response to estrogen therapy had been resistant to this hormone treatment immediately before chemotherapy. Similar observations have been reported after docetaxel and prednisolone therapy (10).

We describe three patients whose second response to hormone therapy occurred in a very different clinical context to those above. There was no exposure to chemotherapy as the relevant therapeutic intervention was salvage brachytherapy.

\section{MATERIALS AND METHODS}

The records of patients at our institution receiving salvage brachytherapy for recurrent localized androgen independent prostate cancer between 1999 and 2007 were reviewed. Localized relapse was diagnosed with repeat prostate biopsy after consistent PSA rise and no evidence of extraprostatic disease seen on bone scan and pelvic magnetic resonance imaging (MRI).

Patients were eligible for inclusion in this review only if they had previously been treated with conventionally fractionated external beam radiotherapy (EBRT) to 68-72Gy (a dose considered radical at the time of first treatment), had subsequently developed biochemical relapse and had initially responded to hormonal treatment before developing androgen resistance.

As salvage brachytherapy is currently unproven, patients were treated under an investigative protocol approved by the Hospital Ethics Committee and all gave full written informed consent to treatment.

Eleven men aged 54-77 were treated within this protocol. Initial results of efficacy and toxicity have been reported elsewhere (11). Nine patients have subsequently developed a further biochemical relapse and, of these, three were found to have disease which did respond to rechallenge with hormone therapy; these three are reported in detail in this manuscript.

\section{RESULTS}

\section{Patient 1}

A 56 year old man presented with Gleason $3+3$ organ-confined prostate adenocarcinoma and prostate-specific antigen (PSA) of $9.9 \mathrm{ng} / \mathrm{mL}$. He was treated with radical EBRT but achieved a PSA nadir of only $2.0 \mathrm{ng} / \mathrm{mL}$ and by 2.5 years, the PSA had risen to $7.2 \mathrm{ng} / \mathrm{mL}$.

Goserelin was started with a PSA fall to 0.3 $\mathrm{ng} / \mathrm{mL}$, maintained for four years when the PSA rose to $1.4 \mathrm{ng} / \mathrm{mL}$ and to $3.0 \mathrm{ng} / \mathrm{mL}$ after a further two years despite ongoing goserelin. Repeat biopsy found Gleason 4+3 disease in both lobes but MRI indicated no extracapsular disease and a bone scan showed no distant disease.

Goserelin was discontinued and the patient underwent salvage ${ }^{125} \mathrm{I}$ seed brachytherapy to a marginal dose of $60 \mathrm{~Gy}$. The PSA fell to $0.8 \mathrm{ng} / \mathrm{mL}$ 
at three months following treatment but rose to 1.2 $\mathrm{ng} / \mathrm{mL}$ and $3.6 \mathrm{ng} / \mathrm{mL}$ at nine and eighteen months respectively.

Goserelin was recommenced and the PSA became undetectable, remaining so for twelve months after restarting the LHRH agonist. At that point, goserelin was stopped and a policy of intermittent anti-androgen therapy instituted: the PSA remained undetectable for another five months before rising to the current level of $1.1 \mathrm{ng} / \mathrm{mL}$ after a further three months. Reintroduction of goserelin again led to PSA falling to an undetectable level (Figure-1).

\section{Patient 2}

A 67 year old man presented with a PSA of $11.9 \mathrm{ng} / \mathrm{mL}$ and was diagnosed with localized Gleason $5+3$ adenocarcinoma of the prostate. He received radical EBRT to a dose of 70 Gy and achieved a PSA nadir of $<1 \mathrm{ng} / \mathrm{mL}$ at two years.

Repeat biopsy following a PSA rise to 4.5 $\mathrm{ng} / \mathrm{mL}$ three years later yielded recurrent adenocarcinoma of the same grade, and goserelin was instituted with a PSA response to near undetectable levels. Two years later, despite continued goserelin, the PSA rose to $1.1 \mathrm{ng} / \mathrm{mL}$, with rises to $3.3 \mathrm{ng} / \mathrm{mL}$ and 5.6 $\mathrm{ng} / \mathrm{mL}$ after one and two further years respectively. The prostate was palpably hard at the right side (the site of the positive biopsy) but further staging investigations were negative for disease beyond the prostate.

Goserelin was discontinued and salvage ${ }^{125} \mathrm{I}$ seed brachytherapy was delivered to a marginal dose of 60 Gy with subsequent PSA fall over six months to $2.3 \mathrm{ng} / \mathrm{mL}$. Nine months later PSA started rising to a peak of $21.0 \mathrm{ng} / \mathrm{mL}$ eighteen months after brachytherapy with inguinal and iliac lymphadenopathy seen on MRI.

Goserelin was reintroduced; the PSA fell to $8.5 \mathrm{ng} / \mathrm{mL}$ after six months but three months later rose to $21.8 \mathrm{ng} / \mathrm{mL}$. Bicalutamide was added and two months later PSA fell to $6.3 \mathrm{ng} / \mathrm{mL}$. Four months later a further PSA rise to $18.6 \mathrm{ng} / \mathrm{mL}$ occurred, bicalutamide was withdrawn with no beneficial effect and the patient declined both glucocorticoids and estrogens in favor of chemotherapy (Figure-1).

\section{Patient 3}

A 77 year old man was found to have Gleason $4+4$ disease with a PSA of $40 \mathrm{ng} / \mathrm{mL}$ and extracapsular extension but no nodal or distant involvement. He received three months of neoadjuvant bicalutamide (150 mg per day) with a PSA fall to $7.4 \mathrm{ng} / \mathrm{mL}$ before radical EBRT to a dose of $70 \mathrm{~Gy}$. With adjuvant bicalutamide for one year the PSA fell further to 1.6 $\mathrm{ng} / \mathrm{mL}$ and the anti-androgen was discontinued.

At twelve months PSA rose to $10 \mathrm{ng} / \mathrm{mL}$ and goserelin was commenced with a PSA fall to 7.4 $\mathrm{ng} / \mathrm{mL}$ six months later. Further rises to $11.2 \mathrm{ng} / \mathrm{mL}$ and $14.2 \mathrm{ng} / \mathrm{mL}$ after another six and nine months indicated progressive disease. MRI and a bone scan showed no extra-prostatic disease and with biopsy proof of Gleason 5+4 disease goserelin was stopped and brachytherapy delivered to a marginal dose of 60 Gy.

There was no significant change to the PSA at six months $(14.9 \mathrm{ng} / \mathrm{mL})$ but at one year it had risen sharply to $87.5 \mathrm{ng} / \mathrm{mL}$ with the subsequent appearance of metastatic disease. Goserelin was restarted and six months later the PSA had fallen to $23 \mathrm{ng} / \mathrm{mL}$. A further fifteen months later the level remains stable at $25 \mathrm{ng} / \mathrm{mL}$ (Figure-1).

\section{COMMENTS}

There are limited treatment options once recurrent prostate cancer develops androgen independence. Palliative chemotherapy with docetaxel has been shown to improve survival (12) and is commonly instituted for metastatic disease following failure of maximal androgen blockade but is not suitable for all patients, particularly those with poor performance status.

Those patients with no discernible distant disease may receive local salvage treatment such as brachytherapy or cryotherapy. Salvage brachytherapy has been shown to deliver a biochemical response in patients with localized disease (13). The dose for patients who have previously received EBRT is necessarily lower than for those who have been treated without radiotherapy and is the subject of debate: whilst many clinicians treat to a marginal dose of 
A

B
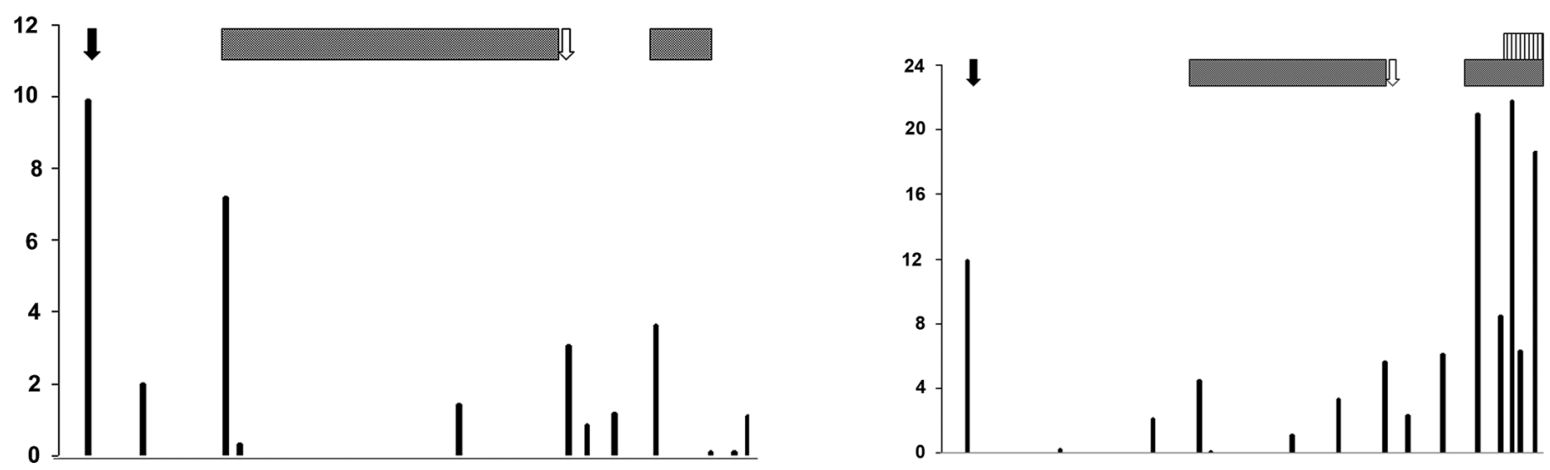

C

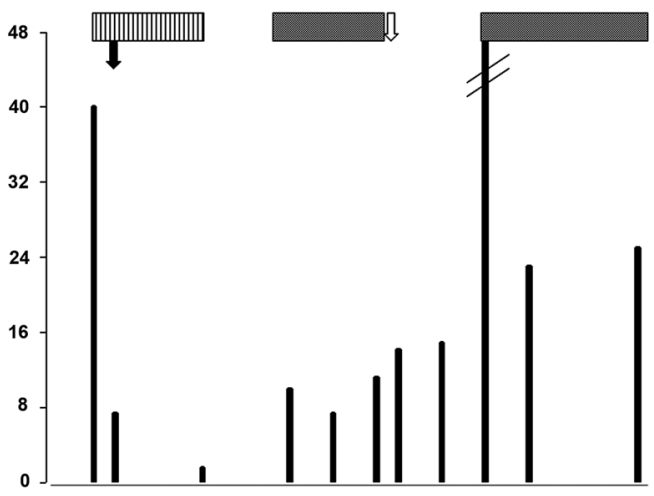

$\downarrow$ = External Beam Radiotherapy

In $=$ Salvage Brachytherapy

$=$ Duration of LHRH agonist

血向而= Duration of antiandrogen

Figure 1 - PSA level according to therapy. For time details see text. A) Patient 1, B) Patient 2, C) Patient 3.

95-100 Gy our practice is to treat to 60 Gy using low dose rate ${ }^{125} \mathrm{I}$ seeds as this has a similar biochemical effect with a lower risk of late toxicity.

The apparent recovery of hormone sensitivity described in these three patients after brachytherapy is an unexpected finding. PSA measurement has long been accepted as a surrogate marker for disease activity as potentially more direct measures such as circulating tumor cell assays remain experimental. The phenomenon of PSA bounce (that is a transient PSA elevation following therapy) is recognized but is unlikely to be relevant to these cases: the phenomenon has not been recorded following salvage brachytherapy after previous EBRT; neither the double response to hormones in case 1 nor the response of metastatic disease in case 3 could be so explained.
We believe the data support tumor progression after brachytherapy, particularly given the magnitude of PSA rise in each case. Moreover, there is the precedent for the reacquisition of hormone sensitivity in the reports following chemotherapy quoted above $(9,10)$.

All three patients had convincing biochemical evidence of androgen independent prostate cancer but after salvage brachytherapy and subsequent biochemical failure were found to have reacquired hormone responsive disease. So good was the response in one patient (Patient 1) that the practice of intermittent endocrine therapy has been introduced. In another patient (Patient 2) the response was complete but brief and in a third (Patient 3), although the response was partial, it was remarkably durable. 
Activity of the androgen receptor is key to regulation of prostate cancer and may be critical to the explanation of this observation (14). Normally, in the absence of ligand, the androgen receptor is held inactive in the cytoplasm by heat shock protein 90 . Testosterone enters the prostate cell and is converted by the enzyme 5 -alpha reductase to its derivative dihydrotestosterone which binds to the androgen receptor causing dimerisation. The receptor then enters the nucleus where binding at androgen responsive elements within regulatory genes, modulated by co-activators and co-repressors, causes increased transcription and cellular proliferation.

Although rarely mutated in localized disease, most androgen insensitive cell lines do show abnormalities of the gene coding for the androgen receptor including gene amplification (15), increased sensitivity to ligand (16) and inappropriate activation by other ligands (17). Thus, in a prostate cancer cell with one or more of these mutations the drive to proliferation persists despite an undetectable level of circulating testosterone.

Furthermore, mutations are known to accumulate during the life of a malignant cell and may result in a particular treatment actually becoming a stimulus for disease progression. For example it was observed that in approximately one third of patients with progression on anti-androgens, withdrawal of that treatment would lead to a PSA fall (18). Subsequently it was shown that amino acid substitutions allowed the receptor to be activated by cortisol, other steroids and even anti-androgens such as flutamide (19). Hence, withdrawal of a previously active treatment may have a beneficial effect.

The cases presented here and in previous reports indicate that the observed clinical state of androgen independence is not necessarily permanent. Whilst many of the mechanisms by which androgen dependence is lost are understood, the mechanisms by which androgen dependence is restored are uncertain.

One explanation could be that the androgen receptor itself has a degree of plasticity and that hormone sensitivity is reinduced by a mechanism perhaps triggered by a therapeutic insult. Chemotherapy has been proposed as that therapeutic insult (9) but our data suggest radiation exposure may similarly act to reverse androgen independence. Several mutations have been outlined above and it is plausible that, were further mutations to take place disabling the abnormal androgen receptor gene and hence the mechanism by which androgen resistance had developed, hormone sensitivity could be regained. This mutation may be induced by radiotherapy or potentially even arise purely from the passage of time: certainly the pelvic side wall metastases in case 2 did not receive brachytherapy.

Alternatively, androgen independent and dependent clones may coexist within a clinical cancer. The measured PSA would reflect PSA production from each clone, changes in PSA would reflect progression or response to treatment of each clone and would be most influenced by the behavior of the dominant clone. Chemotherapy and radiotherapy could act to more selectively deplete androgen independent clones, allowing residual androgen sensitive clones to become the more dominant producers of PSA, and hence the clinical cancer phenotype would seem to return to a hormone sensitive state.

A further explanation may be found in changes to downstream survival pathways, either induced by therapeutic interventions or occurring as de novo mutations. Non-androgen receptor activation pathways have been described including p53 (20) and bcl-2 (21) and disruption to these could affect tumor activity. It is important that subsequent investigations probe whether interruption to these pathways may be helpful.

Such studies may impact on the current trend towards intermittent androgen blockade in the long term care of hormone responsive metastatic prostate cancer. The rationale underlying this strategy is that continuous androgen suppression may produce a natural selection pressure in favor of androgen independent clones whereas intermittent suppression allows cytoreduction during treatment but not to the extent that insensitive clones "outcompete" sensitive clones when treatment ceases. Although clinically attractive, not least owing to presumed reduction of side effects during periods off treatment, there is not yet convincing evidence favoring the intermittent strategy and a large international trial for patients with metastatic disease is currently addressing this question following encouraging preliminary results (22). 


\section{CONCLUSIONS}

The data presented here reconfirm that prostate cancer cells which have developed resistance to androgen deprivation and androgen receptor antagonism may later reacquire sensitivity to that same hormonal therapy. The data demonstrate that this phenomenon is not peculiar to patients who have received cytotoxic chemotherapy but may occur after brachytherapy, either causally related to the brachytherapy or with the passage of time.

These findings suggest that important undetermined mechanisms underlie androgen resistance and give hope that there may be therapeutic interventions available to a large cohort of patients with recurrent prostate cancer previously considered permanently androgen independent, and provoke thought as to whether the policy of intermittent androgen therapy for metastatic prostate cancer may have advantages not previously contemplated.

\section{ACKNOWLEDGEMENTS}

We thank Dr. Jonathan Shamash for reviewing this paper.

\section{CONFLICT OF INTEREST}

None declared.

\section{REFERENCES}

1. Berger R, Febbo PG, Majumder PK, Zhao JJ, Mukherjee S, Signoretti S, et al.: Androgen-induced differentiation and tumorigenicity of human prostate epithelial cells. Cancer Res. 2004; 64: 8867-75.

2. Huggins C: Effect of orchiectomy and irradiation on cancer of the prostate. Ann Surg. 1942; 115: 1192-200.

3. Allen JM, O’Shea JP, Mashiter K, Williams G, Bloom SR: Advanced carcinoma of the prostate: treatment with a gonadotrophin releasing hormone agonist. Br Med J. (Clin Res Ed). 1983; 286: 1607-9.

4. Sogani PC, Whitmore WF Jr: Experience with flutamide in previously untreated patients with advanced prostatic cancer. J Urol. 1979; 122: 640-3.
5. Crawford ED, Eisenberger MA, McLeod DG, Spaulding JT, Benson R, Dorr FA, et al.: A controlled trial of leuprolide with and without flutamide in prostatic carcinoma. N Engl J Med. 1989; 321: 419-24. Erratum in: N Engl J Med. 1989; 321: 1420.

6. Huggins $\mathrm{C}$, Hodges CV: Studies on prostatic cancer, effect of castration, of estrogen and of androgen injection on serum phosphatases in metastatic carcinoma of the prostate. Cancer Res. 1941; 1: 293-7.

7. Plowman PN, Perry LA, Chard T: Androgen suppression by hydrocortisone without aminoglutethimide in orchiectomised men with prostatic cancer. Br J Urol. 1987; 59: 255-7.

8. Attard G, Reid AH, Yap TA, Raynaud F, Dowsett M, Settatree S, et al.: Phase I clinical trial of a selective inhibitor of CYP17, abiraterone acetate, confirms that castration-resistant prostate cancer commonly remains hormone driven. J Clin Oncol. 2008; 26: 4563-71.

9. Shamash J, Davies A, Ansell W, Mcfaul S, Wilson P, Oliver T, et al.: A phase II study investigating the re-induction of endocrine sensitivity following chemotherapy in androgen-independent prostate cancer. Br J Cancer. 2008; 98: 22-4.

10. Cox RA, Sundar S: Re-induction of hormone sensitivity to diethylstilboestrol in androgen refractory prostate cancer patients following chemotherapy. $\mathrm{Br}$ J Cancer. 2008; 98: 238-9.

11. Smith D, Maclean J, Plowman PN. Salvage Iodine125 Brachytherapy for Locally Recurrent Prostate Cancer after External Beam Radiotherapy. Clin Onc (RCR). 2009; 21: 249. Abstract \# 24.

12. Tannock IF, de Wit R, Berry WR, Horti J, Pluzanska A, Chi KN, et al.: Docetaxel plus prednisone or mitoxantrone plus prednisone for advanced prostate cancer. N Engl J Med. 2004; 351: 1502-12.

13. Grado GL, Collins JM, Kriegshauser JS, Balch CS, Grado MM, Swanson GP, et al.: Salvage brachytherapy for localized prostate cancer after radiotherapy failure. Urology. 1999; 53: 2-10.

14. Debes JD, Tindall DJ: Mechanisms of androgen-refractory prostate cancer. N Engl J Med. 2004; 351: 1488-90.

15. Koivisto P, Kononen J, Palmberg C, Tammela T, Hyytinen E, Isola J, et al.: Androgen receptor gene amplification: a possible molecular mechanism for androgen deprivation therapy failure in prostate cancer. Cancer Res. 1997; 57: 314-9.

16. Gregory CW, Johnson RT Jr, Mohler JL, French FS, Wilson EM: Androgen receptor stabilization in recurrent prostate cancer is associated with hypersensitivity to low androgen. Cancer Res. 2001; 61: 2892-8. 
17. Taplin ME, Bubley GJ, Shuster TD, Frantz ME, Spooner AE, Ogata GK, et al.: Mutation of the androgen-receptor gene in metastatic androgen-independent prostate cancer. N Engl J Med. 1995; 332: 1393-8.

18. Kelly WK, Scher HI: Prostate specific antigen decline after antiandrogen withdrawal: the flutamide withdrawal syndrome. J Urol. 1993; 149: 607-9.

19. Monge A, Jagla M, Lapouge G, Sasorith S, Cruchant M, Wurtz JM, et al.: Unfaithfulness and promiscuity of a mutant androgen receptor in a hormone-refractory prostate cancer. Cell Mol Life Sci. 2006; 63: 487-97.

20. Bookstein R, MacGrogan D, Hilsenbeck SG, Sharkey F, Allred DC: p53 is mutated in a subset of advanced- stage prostate cancers. Cancer Res. 1993; 53: 336973.

21. Colombel M, Symmans F, Gil S, O'Toole KM, Chopin $\mathrm{D}$, Benson M, et al.: Detection of the apoptosis-suppressing oncoprotein bc1-2 in hormone-refractory human prostate cancers. Am J Pathol. 1993; 143: 390-400.

22. Albrecht W, Collette L, Fava C, Kariakine OB, Whelan P, Studer UE, et al.: Intermittent maximal androgen blockade in patients with metastatic prostate cancer: an EORTC feasibility study. Eur Urol. 2003; 44: 50511.
Accepted after revision:

December 2, 2009

\section{Correspondence address:}

Dr. P. Nick Plowman

Department of Radiotherapy

St Bartholomew's Hospital

West Smithfield, London, EC1A-7BE

Fax: + 4420 7601-8364

E-mail: nick.plowman@bartsandthelondon.nhs.uk

\section{EDITORIAL COMMENT}

This report of cases is important to urology and oncology. The authors relate recovery of hormone sensitivity after salvage brachytherapy for hormone independent localized prostate cancer. The same phenomenon was observed before chemotherapy. The natural history of prostate cancer, i.e., hormonal sensitive replaced by insensitive cells followed by final progression can be modified. A doubt persists: can this phenomenon be applied to disseminated disease? I would like to congratulate the authors and suggests renaming this effect to "insensitive-to-sensitive hormonal retroconvertion of prostate cancer".

Dr. Daniel Seabra

Head, Urologic Oncology

Santo Amaro University

Sao Paulo, SP, Brazil

E-mail: daniel.seabra@terra.com.br 


\section{EDITORIAL COMMENT}

The use of PSA as an effective marker of clinical success in the absence of measurable disease has been a boon to the evaluation of prostate cancer therapies. The selection of further treatment after a definitive therapy depends on many factors and treatment goals are prolonging survival, preventing or delaying symptoms due to disease progression, improving and maintaining quality of life, reducing treatment related morbidity.

In the past, only patients with proven metastatic disease or those with post-local therapy failures received hormonal treatment, being the androgen suppression therapy considered a mainstay of treatment only for men with advanced prostate cancer. Recent demographic changes in patients treated with hormonal therapy now include not only the patients previously mentioned, but also patients with biochemical failures, those on intermittent therapy, those at high risk for recurrence (T3-4, Gleason score $\geq 8$, PSA $>20$ ), and patients with locally advanced disease treated with radiation. Thus, the extent of disease at the time of hormonal therapy initiation and ultimate hormone refractoriness may vary considerably.

However, it is not clear whether early androgen suppression for men with locally advanced disease or asymptomatic metastases improves length and quality of life compared to androgen suppression deferred until signs and symptoms of clinical progression. The significance of prostate-specific antigen increases during the recovery of androgen after androgen deprivation therapy and radiotherapy for prostate cancer is not well understood, being a matter of intensive investigation.

An occasional patient can be salvaged with prostatectomy after a local recurrence following definitive radiation therapy; however, only about $10 \%$ of patients treated initially with external radiation therapy will have local relapse only. In these patients, prolonged disease control is often possible with hormonal therapy, with median cancer-specific survival of 6 years after local failure $(1,2)$. Despite initial success with hormonal therapy, the durability of this response (median duration $<2$ years) is inadequate, and subsequent treatment is needed for these patients. Patients with rising prostate-specific antigen (PSA) but with castrate testosterone levels still may be susceptible to hormonal therapy. If the patient is not taking an antiandrogen it should be prescribed. If the patient is already taking an antiandrogen, it should be withdrawn. Some studies have shown that withdrawal of antiandrogen may led a fall in PSA level, this is called "antiandrogen withdrawal syndrome", and its due to a mutant androgen receptor in which antiandrogen was thought to actually stimulate cell growth (3). Thus, when a rise in PSA level occurs in a patient taking an antiandrogen, the antiandrogen use should be discontinued and the patient's PSA level should be followed. Once both, antiandrogen therapy and withdrawal have been utilized, the next step is the suppression of adrenal hormones, that can account for $10 \%$ of circulating testosterone. Ketoconazole, aminoglutethimide, and hydrocortisone can suppress its production. Prostate cancer should be considered as hormonal refractory only when all of the above maneuvers have failed.

This study sought to determine whether salvage brachytherapy for localized (androgen independent prostate cancer) after previous external beam radiotherapy could lead to return of hormone sensitivity. Despite the small number of patients evaluated, doses levels inferior to what is recommended when using modern techniques of radiotherapy (Significant clinical data are available demonstrating that dose escalation radiation therapy has a significantly better outcome as the dose to the prostate is increased (4)), and patients not being accrued into any investigative protocol, the paper shows that there is a field and new possibilities for re-irradiation, with either external beam or brachytherapy.

The use of permanent (low dose rate) or temporary (high dose rate) brachytherapy after a local failure is a strategy that can to be added to the treatment arsenal, allowing both, local control and or hormone sensitivity recovery, but its indication is highly dependent on previous dose administered and toxicity reported and expected.

The salvage therapy field is an open book and patients should be advised of expected outcomes. As 
an investigational procedure it should be considered ideally in a clinical trial, particularly if the patient has good prognostic factors (e.g., performance status and hemoglobin level).

\section{REFERENCES}

1. Moul JW, Paulson DF: The role of radical surgery in the management of radiation recurrent and large volume prostate cancer. Cancer. 1991; 68: 1265-71.

2. Schellhammer PF, Kuban DA, el-Mahdi AM: Treatment of clinical local failure after radiation therapy for prostate carcinoma. J Urol. 1993; 150: 1851-5.
3. Taplin ME, Bubley GJ, Ko YJ, Small EJ, Upton M, Rajeshkumar B, et al.: Selection for androgen receptor mutations in prostate cancers treated with androgen antagonist. Cancer Res. 1999; 59: 2511-5.

4. Symon Z, Griffith KA, McLaughlin PW, Sullivan M, Sandler HM: Dose escalation for localized prostate cancer: substantial benefit observed with 3D conformal therapy. Int J Radiat Oncol Biol Phys. 2003; 57: 384-90.

Dr. Antonio Cassio Assis Pellizzon

Head, Brachytherapy Service AC Camargo Hospital \& Prostate Institute Oswaldo Cruz German Hospital São Paulo, SP, Brazil E-mail: cassiopellizzon@aol.com 